\title{
Práticas de autocuidado e os efeitos colaterais imediatos em mulheres com câncer
}

\section{ginecológico em braquiterapia*}

Self-care practices and immediate side effects in women with gynecological cancer in brachytherapy

Prácticas de autocuidado y los efectos colaterales inmediatos en mujeres con cáncer ginecológico en

braquiterapia

\section{Rosimeri Helena da Silva ${ }^{\mathrm{I}}$, Luciana Martins da Rosa ${ }^{\mathrm{II}}$, Mirella Dias ${ }^{\mathrm{III}}$, Nádia Chiodelli Salum ${ }^{\mathrm{IV}}$, Ana Inêz Severo Varelav, Vera Radünz ${ }^{\mathrm{vI}}$}

\begin{abstract}
Resumo: Objetivo: revelar os efeitos colaterais imediatos e as práticas de autocuidado adotadas por mulheres com câncer ginecológico submetidas à braquiterapia. Método: pesquisa narrativa, realizada com 12 mulheres, no Sul do Brasil, entre dezembro/2018 e janeiro/2019, incluindo entrevista semiestruturada submetida à análise de conteúdo. Resultados: da análise emergiram três categorias temáticas: Cuidados orientados e adotados pelas mulheres em braquiterapia pélvica; Efeitos colaterais imediatos percebidos pelas mulheres em braquiterapia pélvica; Cuidados não orientados pelos profissionais da saúde. Os cuidados orientados pelas enfermeiras mais relatados pelas mulheres foram dilatação vaginal, uso de ducha e lubrificante vaginal, consumo de chá, higienização e guarda do dilatador vaginal. Os efeitos colaterais mais citados nas entrevistas foram alterações urinárias e intestinais na pele e mucosas. Conclusão: evidencia-se que a atenção de enfermagem em braquiterapia deve priorizar cuidados para prevenir e controlar as alterações geniturinárias e tegumentares, incluindo práticas de autocuidado.
\end{abstract}

I Mestre Profissional em Gestão do Cuidado em Enfermagem, Enfermeira do Centro de Pesquisas Oncológicas, Florianópolis, Santa Catarina, Brasil. E-mail: merinhahs_@hotmail.com, Orcid: https://orcid.org/0000-0003-1286-567X

II Doutora em Enfermagem, Professora do Programa de Pós-Graduação Gestão do Cuidado de Enfermagem da Universidade Federal de Santa Catarina, Florianópolis, Santa Catarina, Brasil. E-mail: luciana.m.rosa@ufsc.br, Orcid: https://orcid.org/0000-0002-1884-5330

III Doutora em Ciências Médicas, Professora da Universidade do Estado de Santa Catarina, Fisioterapeuta do Centro de Pesquisas Oncológicas, Florianópolis, Santa Catarina, Brasil. E-mail: mirelladias.fisio@gmail.com, Orcid: https://orcid.org/0000-0002-2109-3563

IV Doutora em Enfermagem, Professora do Programa de Pós-Graduação Gestão do Cuidado de Enfermagem da Universidade Federal de Santa Catarina, Enfermeira do Hospital Universitário Polydoro Ernani de São Thiago, Florianópolis, Santa Catarina, Brasil. E-mail: mchiodelli@gmail.com, Orcid: https://orcid.org/0000-0002-2624-6477

V Mestre Profissional em Gestão do Cuidado em Enfermagem, Enfermeira do Centro de Pesquisas Oncológicas, Florianópolis, Santa Catarina, Brasil. E-mail: ainezmpenf@gmail.com, Orcid: https://orcid.org/0000-0002-6221-582X

VI Doutora em Enfermagem, Professora do Departamento de Enfermagem da Universidade Federal de Santa Catarina, Florianópolis, Santa Catarina, Brasil. E-mail: vera.radunz@ufsc.br, Orcid: https://orcid.org/0000-0002-9262-8457

* Extraído da dissertação "Cartilha educativa às mulheres com câncer ginecológico submetidas à braquiterapia”, Programa de Pós-Graduação Gestão do Cuidado em Enfermagem - Modalidade Profissional, Universidade Federal de Santa Catarina, 2020. 
Práticas de autocuidado e os efeitos colaterais imediatos em mulheres com câncer... $\mid 2$

Descritores: Oncologia; Enfermagem; Braquiterapia; Neoplasias dos genitais femininos; Narrativa pessoal

\begin{abstract}
Objective: to reveal the immediate side effects and self-care practices adopted by women with gynecological cancer submitted to brachytherapy. Method: narrative research, conducted with 12 women, in southern Brazil, between December/2018 and January/2019, including semi-structured interviews submitted to content analysis. Results: three thematic categories emerged from the analysis: Care oriented and adopted by women in pelvic brachytherapy; Immediate side effects perceived by women in pelvic brachytherapy; Care not guided by health professionals. The care provided by the nurses most reported by the women was vaginal dilation, use of a shower and vaginal lubricant, tea consumption, cleaning, and storage of the vaginal dilator. The side effects most frequently mentioned in the interviews were urinary and intestinal changes in the skin and mucous membranes. Conclusion: nursing care in brachytherapy must prioritize care to prevent and control genitourinary and cutaneous changes, including self-care practices.
\end{abstract}

Descriptors: Oncology; Nursing; Brachytherapy; Neoplasms of the female genitals; Personal narrative

Resumen: Objetivo: revelar los efectos colaterales inmediatos y las prácticas de autocuidado adoptadas por mujeres con cáncer ginecológico sometidas a la braquiterapia. Método: investigación narrativa, realizada con 12 mujeres, en el Sur de Brasil, entre diciembre/2018 y enero/2019, incluyendo entrevista semiestructurada sometida al análisis de contenido. Resultados: del análisis surgieron tres categorías temáticas: Cuidados orientados y adoptados por las mujeres en braquiterapia pélvica; Efectos colaterales inmediatos percibidos por las mujeres en braquiterapia pélvica; Cuidados no orientados por los profesionales de la salud. Los cuidados orientados por las enfermeras más relatados por las mujeres fueron dilatación vaginal, uso de ducha y lubrificante vaginal, consumo de té, higienización y guarda del dilatador vaginal. Los efectos colaterales más citados en las entrevistas fueron alteraciones urinarias e intestinales en la piel y mucosas. Conclusión: se observa que la atención de enfermería en braquiterapia debe priorizar cuidados para prevenir y controlar las alteraciones genitourinarias y pigmentarias, incluyendo prácticas de autocuidado.

Descriptores: Oncología; Enfermería; Braquiterapia; Neoplasias de los genitales femeninos; Narrativa personal

\title{
Introdução
}

Os cânceres ginecológicos, excluindo o câncer de mama, acometem o colo e o corpo do útero (destacando-se o endométrio), os ovários, as tubas uterinas, a vagina e a vulva. As três primeiras topografias citadas destacam-se por suas incidências, mortalidades e prevalências. Estima-se que as elevadas incidências dessas doenças persistam nas próximas décadas, principalmente nos países em desenvolvimento e subdesenvolvidos. Atualmente, a incidência mundial do câncer do colo do útero, do corpo do útero e dos ovários, equivale, respectivamente, a $569.847,382.069$ e a 295.414 casos novos. ${ }^{1-3}$

A cirurgia, quimioterapia, radioterapia e hormonioterapia são terapêuticas largamente utilizadas para o controle dos cânceres ginecológicos. A braquiterapia, uma das principais 
modalidades de tratamento para os cânceres ginecológicos, configura uma forma específica de radioterapia (aplicação de radiação ionizante), que consiste na colocação precisa de fontes radioativas dentro ou próximas ao tumor. ${ }^{4-5}$

Para o tratamento do câncer do colo do útero localmente avançado, o tipo de câncer ginecológico mais incidente, há a indicação da administração simultânea de teleterapia (radioterapia externa) com quimioterapia, seguida da braquiterapia (radioterapia interna). A associação da braquiterapia com outras terapêuticas é fator contribuinte para o surgimento de efeitos colaterais. ${ }^{5}$

Os efeitos colaterais decorrentes da braquiterapia podem ser imediatos ou tardios. Os principais efeitos colaterais físicos imediatos incluem inflamação da mucosa vaginal, diarreia, sangramento vaginal, fadiga e alteração vesical. Os efeitos colaterais tardios mais comuns são sangramento vaginal, ulcerações retais, alterações vesicais, encurtamento e estreitamento da vagina (estenose vaginal). Registra-se que os efeitos tardios se iniciam, geralmente, após três meses do término da braquiterapia ou durante os três primeiros anos. ${ }^{6-7}$

A adoção de cuidados em saúde pode prevenir ou minimizar esses efeitos colaterais. Assim, a consulta de enfermagem com foco na educação em saúde para o autocuidado, centrada nas necessidades de cada mulher, deve ser iniciada antes mesmo do início do tratamento. $\mathrm{O}$ processo educativo deve ter continuidade durante e após o término da braquiterapia, de forma a minimizar os possíveis danos relacionados à saúde da mulher. ${ }^{8-9}$

No Brasil, a adesão das mulheres, submetidas à braquiterapia, às práticas de autocuidado mostra-se aquém das necessidades em saúde, assemelhando-se às dificuldades evidenciadas em outros países. A definição de recomendações específicas às mulheres, padronizadas pelos serviços, a partir das evidências científicas, quantitativas e/ou qualitativas, favorece melhores resultados observados na prática clínica. ${ }^{8-9}$

No Ambulatório de Radioterapia do Centro de Pesquisas Oncológicas (CEPON) (Santa Catarina/Brasil), as mulheres em braquiterapia pélvica são orientadas pelas enfermeiras nas 
Práticas de autocuidado e os efeitos colaterais imediatos em mulheres com câncer... 4

consultas de enfermagem, realizadas antes, durante e ao término da braquiterapia. Nessas consultas, as enfermeiras visam ao acolhimento, aos esclarecimentos sobre o tratamento e efeitos colaterais e aos cuidados a serem adotados durante e após término da braquiterapia. Concluído o tratamento, as mulheres permanecem em seguimento periódico com a fisioterapeuta, além das consultas médicas com o radioterapeuta e oncologista.

Na perspectiva de aprimorar a educação em saúde desenvolvida nas consultas de enfermagem, as enfermeiras do CEPON observaram a necessidade de construção de uma cartilha educativa para auxiliar às mulheres na adoção de cuidados durante e após a braquiterapia. Para favorecer essa construção, idealizou-se o objeto desta investigação, tendo como questão de pesquisa: quais os efeitos colaterais imediatos percebidos por mulheres com câncer ginecológico durante a braquiterapia e as práticas de autocuidado orientadas pela equipe e adotadas por essas mulheres?

A inclusão de material educativo constitui-se como uma boa prática, pois complementa as ações de educação em saúde ofertadas nas consultas de enfermagem e auxilia a compreensão e adesão ao tratamento. Mulheres em braquiterapia bem informadas e aconselhadas são menos propensas a ter sentimentos, como o medo e a ansiedade, sendo mais provável que sua experiência terapêutica seja lembrada como positiva. ${ }^{10}$

Nesse contexto assistencial, entende-se que a identificação dos efeitos colaterais imediatos, percebidos pelas próprias mulheres, e os cuidados que são realizados por elas, após as orientações profissionais, podem contribuir para a avaliação de enfermagem e construção dos conteúdos de cartilha educativa, complementando a educação em saúde iniciada na consulta de enfermagem. Assim, justifica-se o desenvolvimento deste estudo.

Ainda se registra que a literatura científica aponta os efeitos colaterais imediatos e tardios que podem ocorrer em consequência da braquiterapia pélvica. O tipo de aparelho utilizado para administração da radiação ionizante contribui para o melhor controle e prevenção desses efeitos colaterais, porém a abordagem das enfermeiras também configura outro fator 
contribuinte. ${ }^{8-9,11}$ Mas, quando a abordagem clínica é pautada nas necessidades das mulheres, ela tende a ampliar os resultados de enfermagem.

Nessa perspectiva, a contribuição desta investigação vincular-se-á às ações de enfermagem pautadas nas necessidades em saúde reveladas pelas mulheres com câncer ginecológico em braquiterapia, que são abordadas nas consultas de enfermagem, conforme afirmações da equipe, e que serão incluídas na cartilha educativa a ser construída. Portanto, objetiva-se revelar os efeitos colaterais imediatos e as práticas de autocuidado adotadas por mulheres com câncer ginecológico submetidas à braquiterapia.

\section{Método}

Trata-se de pesquisa narrativa, uma metodologia que permite a coleta de histórias sobre um determinado fenômeno e a compreensão da experiência vivida, em um processo que inclui a colaboração do pesquisador e do participante para apreensão do vivido. No momento da aplicação das entrevistas na coleta dos dados, neste tipo de investigação, é recomendado para a narração central do participante o uso de pergunta disparadora, sendo que o pesquisador não deve interromper a narração, somente deve encorajar a continuidade do relato utilizando a comunicação não verbal. Perguntas complementares podem ser realizadas para ampliar a narrativa, desde que o pesquisador não emita opiniões e não discuta sobre contradições. ${ }^{12}$ Associada a essa metodologia, adotou-se a técnica de análise de conteúdo ${ }^{13}$ para a compreensão e apresentação do fenômeno investigado.

O cenário do estudo foi o Ambulatório de Radioterapia do CEPON, que atende cerca de dez mulheres a cada mês; $79 \%$ delas com diagnósticos de câncer do colo do útero e 19\% do endométrio. ${ }^{14}$ Registra-se que esse cenário de atenção oncológica oferta, desde 2006, a braquiterapia de alta taxa de dose às mulheres com cânceres ginecológicos, que consiste na aplicação da radiação ionizante pela fonte Irídio 192 e por um conjunto de procedimentos realizados pela equipe de saúde para maior segurança da mulher. De acordo com as 
Práticas de autocuidado e os efeitos colaterais imediatos em mulheres com câncer... 6

recomendações internacionais, a dose total de tratamento para a braquiterapia de alta taxa de dose é de 25 a 30 Gray, sendo administradas até cinco frações separadas, no máximo duas frações por semana e nunca em dias consecutivos. ${ }^{11}$ No ambulatório do CEPON, a braquiterapia é administrada às mulheres com câncer ginecológico em três (histerectomizadas) ou quatro (não histerectomizadas) frações, em até 15 dias.

Incluíram-se na investigação mulheres com câncer ginecológico, submetidas à braquiterapia, retornando ao serviço para consulta de seguimento com o radioterapeuta, cerca de 45 dias após o término do tratamento. Definiram-se como critérios de exclusão mulheres com relato de dor e alterações clínicas que dificultassem a comunicação durante a coleta de dados, por exemplo, náuseas intensas e nível de consciência alterado. Todas as mulheres selecionadas e atendidas no período da coleta de dados foram consideradas elegíveis, pois atenderam aos critérios de inclusão. Realizou-se o convite para participação ao término da consulta médica.

O período da coleta dos dados ocorreu entre os meses de dezembro de 2018 e janeiro de 2019. Todas as pacientes agendadas nesse período (12 mulheres) foram selecionadas e incluídas na investigação. O número de participantes foi definido pela saturação dos dados, identificada quando novas informações não foram encontradas nas narrativas das mulheres no desenvolvimento da análise de conteúdo, ${ }^{13}$ o que definiu a conclusão do período de coleta de dados.

Para coleta dos dados, utilizou-se a técnica de entrevista semiestruturada, aplicada por enfermeira atuante no cenário do estudo e pesquisadora principal deste estudo. Elas foram realizadas em consultório de enfermagem, após a consulta com o radioterapeuta, com atenção à privacidade das participantes. Tiveram a duração aproximada de 25 minutos, foram gravadas e transcritas mediante autorização obtida na aplicação e assinatura do Termo de Consentimento Livre e Esclarecido.

As perguntas fechadas abrangeram a idade das participantes, o diagnóstico do câncer, estado marital, uso do dilatador vaginal (dispositivo de silicone, no formato de pênis, ofertado pela enfermeira às mulheres, para dilatação vaginal após braquiterapia, em geral, nominado pelas 
enfermeiras do CEPON e pacientes por prótese vaginal) e manutenção da relação sexual. Enquanto as perguntas abertas, consideradas disparadoras para obtenção das narrativas, foram "Você poderia contar quais as reações, ou os efeitos colaterais, que ocorreram com você durante a braquiterapia? Quais os cuidados que foram orientados pelas enfermeiras e outros profissionais que você realizou ou está realizando? Quais cuidados você indica serem abordados nas consultas de enfermagem e incluídos na cartilha educativa que será construída pelas enfermeiras do CEPON e que serão entregues às mulheres que vão iniciar a braquiterapia? Você teria alguma outra recomendação?

As comunicações obtidas foram submetidas à análise de conteúdo,${ }^{13}$ assim, após leitura exaustiva, identificaram-se as unidades de registros. Os códigos das unidades de registros foram definidos por termos representativos dos significados expressos. Regras de enumeração foram aplicadas nos dados investigados nas perguntas fechadas.

Sequencialmente, agruparam-se as unidades de registros por semelhança para construção das categorias temáticas. A etapa de codificação e categorização das comunicações foi realizada por duas codificadoras entre março e maio de 2019. Para interpretação e inferência dos dados, realizou-se análise reflexiva e crítica dos resultados obtidos, sustentada por conhecimentos científicos atualizados e publicados no meio científico sobre a temática em questão.

Este estudo foi conduzido de acordo com os padrões éticos exigidos pela Resolução 466/2012 do Conselho Nacional de Saúde. A aprovação ética para seu desenvolvimento encontrase registrada sob os pareceres $n^{\circ} 2730286$ (proponente - data de aprovação 22/06/2018) e $n^{\circ}$ 2798839 (coparticipante - data de aprovação 03/08/2018). O anonimato das participantes foi garantido por codificação alfanumérica - a sigla MSB (Mulher Submetida à Braquiterapia), seguida de um número arábico sequencial (MSB1 até MSB12).

\section{Resultados}

As inclusões totalizaram 12 mulheres, com idades entre 33 e 74 anos (média de 54 anos), 
Práticas de autocuidado e os efeitos colaterais imediatos em mulheres com câncer... $\mid 8$

sendo sete com diagnóstico de câncer de colo do útero e cinco do endométrio; a maioria casada ou em união estável (oito mulheres). Quanto ao uso do dilatador vaginal e manutenção da relação sexual, todas as participantes referiram usar duas a três vezes por semana o dilatador, oito afirmaram não manter relações sexuais.

Da análise de conteúdo emergiram três categorias temáticas, a saber: Cuidados orientados e adotados pelas mulheres em braquiterapia pélvica; Efeitos colaterais imediatos percebidos pelas mulheres em braquiterapia pélvica; Cuidados não orientados pelos profissionais da saúde. Essas categorias temáticas são apresentadas sequencialmente.

\section{Cuidados orientados e adotados pelas mulheres em braquiterapia pélvica}

Esta categoria temática constituiu-se dos relatos das mulheres verbalizando os cuidados orientados pelas enfermeiras e outros profissionais da equipe de saúde, no período da braquiterapia pélvica, para adoção nas práticas de autocuidado, durante e após o término do tratamento. Os cuidados orientados pelos enfermeiros e mais relatados pelas mulheres (relatos de seis mulheres) foram: uso do dilatador vaginal; uso da ducha vaginal com aplicação do chá de camomila; consumo de chá de quebra-pedra (hidratação e ação diurética para prevenir alterações urinárias); uso de lubrificante vaginal; higienização e guarda do dilatador vaginal.

Outros cuidados orientados e revelados pelas participantes abrangeram: ingesta hídrica diária de 2 litros ou mais; realização de banho de assento com chá de camomila; manutenção ou interrupção da relação sexual durante a braquiterapia; manutenção da relação sexual após o término do tratamento e seus benefícios; uso de preservativo lubrificado; higienização do corpo antes da braquiterapia; uso de óleo mineral e laxante nos casos de constipação; uso de medicamento tópico para tratamento de lesões da pele e mucosas; uso de supositórios com corticoide; uso de medicamento para controle das ardências urinárias; comunicação das alterações percebidas à equipe de saúde; repouso (na presença da fadiga); realização de atividade 
física diária por 30 minutos, como caminhadas; exposição ao banho de sol por 30 minutos diários (antes das 10 horas e após as 16 horas):

Ah, fui orientada a usar a prótese [...] usava um dia sim, um dia não, e continuei com o banho, [...] porque o médico falou que seria bom fazer mais banho, banho interno com a ducha. Fiz ducha bastante tempo não tive problema nenhum. [...] Eu passo na água fervendo e uso camisinha na prótese peniana [...] tenho que usar a que não tem látex, porque eu tenho alergia ao látex, e uso óleo [se referindo ao uso do lubrificante vaginal]. (MSB1)

Eu fiz chá, fui orientada a fazer chá de camomila e foi tranquilo para manter a relação sexual. Ah, elas [enfermeiras] me deram uma [...] Nem sei te dizer o nome [...]. Elas me deram ducha ginecológica, daí eu botava o chá lá dentro e fazia a lavagem vaginal, ia e voltava bem tranquilo. Também fui orientada que eu ia me sentir bem com o chá, para depois fazer xixi. Assim, me ajudou bastante, eu me sentia bem aliviada. (MSB2)

[...] se tivesse alguma ardência ou diarreia, vômitos, essas coisas era para avisar. [...] Fazer a fisioterapia em casa [...]. Eles me deram a prótese aqui no hospital e eu fiz em casa e faço, às vezes, três vezes por dia. Não estou mantendo a relação sexual, eu estou separada. [...] usava a ducha, já lavo embaixo do chuveiro, ali tudo certinho e boto saquinho e guardo na caixinha de novo e a prótese a mesma coisa, eu lavo embaixo do chuveiro, tem dias que eu passo álcool, tem dias que passo soro. (MSB3)

Bastante hidratação, água? Natural, normal, bastante chá de camomila para fazer banhos, usei bastante também chá de quebra-pedra, para [...] fazer bastante xixi [...] usei a prótese com bastante lubrificação, gel mesmo, usei também bastante os cremes indicados para aliviar o ressecamento da pele. [...] tomei pyridium, porque, realmente o chá, assim, podia ser um pouco mais demorado, continuei com chá, mas tomei uma, duas, três semanas seguidas, e agora eu venho usando supositório, que é corticoide [para tratar lesões no intestino]. (MSB4)

[...] para não fazer serviço pesado em casa, para andar um pouco no sol, meia hora só. (MSB5) 
Práticas de autocuidado e os efeitos colaterais imediatos em mulheres com câncer... |10

Sim eu passo um creme vaginal, aquele " $K Y$ ”. (MSB6)

Camisinha e o gel. Eu lavo [a prótese peniana], guardo, lavo só água normal. Daí, eu coloco no pacote que ela me deu. Eu guardo sempre ali, só uso, lavo e guardo. (MSB7)

Comparando os relatos das participantes com a prática clínica, observou-se que os cuidados orientados e mais adotados pelas mulheres se referem às orientações ofertadas pelos enfermeiros no momento do término da alta do tratamento, exceto o consumo de chá, destacado desde a primeira consulta de enfermagem, no início do tratamento.

\section{Efeitos colaterais imediatos percebidos pelas mulheres em braquiterapia pélvica}

Esta categoria agrupa os efeitos colaterais ocorridos durante e após os 45 dias posteriores ao término da braquiterapia. Os efeitos colaterais que emergiram com maior frequência foram: alterações intestinais, tais como constipação, diarreia, urgência na evacuação; ressecamento e hiperemia da pele; ressecamento vaginal; inflamação da mucosa vaginal, anal e retal; e disúria. Outros efeitos colaterais revelados nas narrativas das participantes abrangeram sangramento vaginal; dor abdominal e na vagina; cólica abdominal durante a retirada dos instrumentais da braquiterapia; anemia (com necessidade de transfusão sanguínea):

[...] dificuldade para fazer coco, eu tenho que estar usando sempre dulcolax e óleo mineral e se eu não tomar eu tenho mal estar horrível e tenho dor na barriga. Fazendo o tratamento com dulcolax e tomando o óleo e um monte verdura e aí eu faço coco. [...] queimou ali no ânus, na vagina [...] o médico me examinou eu tive que usar até uma pomada para poder ficar melhor, porque era muita dor e até sangrava. [...] eu sentia muita dor na hora de tirar o aparelho. (MSB1)

$A$ área fica ressecada [referindo-se à vagina], machuca um pouco, mas é suportável. Na entrada do ânus, um ressecamento, e até umas feridinhas, uns machucadinhos, mas com o tempo a gente vai tratando e vai aliviando 
e ajuda bastante. (MSB4)

Eu tive bastante ardência [urinária] nas primeiras semanas, eu fiz quatro sessões de braquiterapia, na terceira, precisei fazer transfusão de sangue, porque meus leucócitos estavam muito baixos. (MSB5)

$A h$, intestino até hoje praticamente trancou, eu tomo, às vezes, um remedinho, mas [...] vou uma, duas vezes, daí não preciso, mas, às vezes, sinto muita vontade, parece que eu tenho que sair correndo para o banheiro, e chego lá é pouquinho. A parte urinária até está normal, assim, dói um pouco por baixo, sabe? [...] Tenho um pouco de ressecamento na vaginal e dor durante o exercício [de dilatação vaginal]. Até eu falei com $o$ doutor, agora, ele disse que é para usar bastante a prótese, porque já está atrofiando. (MSB6)

Os efeitos colaterais aqui apresentados configuram as principais toxicidades esperadas no contexto de atenção oncológica em braquiterapia, sendo que os cuidados de enfermagem implementados no CEPON são fortemente direcionados para prevenção dessa sintomatologia.

\section{Cuidados não orientados pelos profissionais da saúde}

Esta categoria se constituiu do relato de uma participante que referiu o não recebimento de orientações sobre a percepção dolorosa durante a braquiterapia, a depilação a ser realizada antes do início da braquiterapia e a orientação para dilatação vaginal após o término da braquiterapia. Apresenta-se a seguir o depoimento que revela os cuidados não orientados pelos profissionais na percepção da participante. Outros relatos inexistem:

A única orientação que eu não recebi, que não fiz, foi sobre a depilação. Ai o médico me cobrou. Ninguém me falou nada [...] a única dúvida que eu tive, foi que eu recebi a prótese e não recebi orientação para usar. Daí, fui chamada na fisio [...] eu cheguei, meu Deus [...] como eu vou usar isso agora? Aí, dois dias depois já conversei com a fisio, aí ela me orientou a usar a prótese. E faço deitada normal, pronto, 20 minutos. [...] ela disse que isso é comum [...] então, deveria ter sido orientada, que talvez ia ser um pouquinho mais complicado. [...]. (MSB1) 
Práticas de autocuidado e os efeitos colaterais imediatos em mulheres com câncer... | 12

Com relação ao questionamento sobre quais recomendações deveriam ser abordadas nas consultas de enfermagem e incluídas na cartilha educativa, os relatos de 11 participantes revelaram que as mesmas não tinham novas sugestões, pois consideravam que o orientado pelas enfermeiras do CEPON estava adequado e que a cartilha deveria replicar suas ações. Entretanto, uma participante revelou alguns cuidados que não foram orientados pelas enfermeiras e recomendou que situação semelhante seja evitada.

\section{Discussão}

As idades das participantes oscilaram significativamente, como apresentado nos resultados, no entanto a média das idades (próxima aos 50 anos) assemelha-se às pesquisas realizadas nos Estados Unidos da $A_{\text {mérica }}{ }^{15}$ e no próprio cenário do estudo ${ }^{14}$ que apontaram que aquelas com câncer ginecológico tiveram média de idade, respectivamente, de 55 e 51, 53 anos. O câncer do colo de útero, topografia mais incidente dentre os cânceres ginecológicos, é raro até 30 anos, mas sua incidência aumenta progressivamente na faixa de 45 a 50 anos de idade. ${ }^{15}$

Ainda relativo à variabilidade das idades encontradas, associada à investigação realizada anteriormente no CEPON,${ }^{14}$ identificou-se o diagnóstico da doença em idades precoces e tardias nas mulheres assistidas nesse cenário de atenção, o que reforça a necessidade de programas de rastreamento para detecção precoce das lesões não neoplásicas na atenção básica e na média complexidade.

A realização de rastreamento do câncer de colo do útero, pelo menos uma vez na vida, após os 35 anos, diminui o risco de morrer em 70\%. Se a mulher for rastreada a cada cinco anos, esse risco diminui para $85 \%$. Mas, infelizmente, cerca de 1,5 bilhão de mulheres, em todo o mundo, nunca foi rastreado para câncer de colo do útero. ${ }^{16}$ Tal condição explica a elevada incidência do câncer de colo do útero e mortes relacionadas. Quanto aos diagnósticos das 
neoplasias malignas das participantes, estes replicam os achados de outros estudos, que apontam os casos de câncer do colo do útero e do endométrio como os tipos mais incidentes dentre os cânceres ginecológicos..$^{1,14}$

Ainda discutindo os resultados obtidos com a aplicação das perguntas fechadas das entrevistas, observou-se que o cuidado realizado após braquiterapia, no que se refere à dilatação vaginal, retrata o seguimento das orientações dos enfermeiros. Entretanto, na prática clínica do CEPON até 2015, ainda se observava que algumas mulheres tinham dificuldades para a adesão dessa prática.

Para mudar essa realidade, padronizou-se que as pacientes deveriam consultar periodicamente com a fisioterapeuta (ação instituída no ano de 2016) no seguimento do controle do câncer. Nessa consulta, reafirma-se os benefícios da dilatação vaginal em um diálogo minucioso, complementando os esclarecimentos iniciados pela enfermeira na alta da braquiterapia. Ainda, ocorre a avaliação do assoalho pélvico, a verificação da profundidade vaginal e a classificação da estenose vaginal, entre outros aspectos avaliados. Essa prática serve para conscientização das mulheres no que se refere à importância da prevenção desse efeito colateral.

Estudos apontam que as mulheres têm dificuldades para inclusão dos exercícios de dilatação para prevenção da estenose vaginal de forma continuada. ${ }^{8-9,17}$ Assim, os achados desta investigação diferem dessas outras pesquisas. ${ }^{8-9,17}$ Relaciona-se essa conquista à presença de enfermeiros exclusivos para atuar no Ambulatório de Braquiterapia do CEPON, à realização da consulta de enfermagem antes, durante e ao término da terapêutica e ao seguimento periódico com a fisioterapeuta.

No CEPON, pesquisa realizada com 84 mulheres após a braquiterapia, verificou uma adesão de $41,7 \%$ ao uso do dilatador vaginal no formato de pênis de silicone. A pesquisa ainda mostra que as mulheres que realizaram a dilatação vaginal, conforme o recomendado, tiveram um menor grau de estenose vaginal quando comparadas as que não realizaram. ${ }^{18}$

Nos últimos anos, observou-se, empiricamente, que o percentual de adesão à dilatação 
Práticas de autocuidado e os efeitos colaterais imediatos em mulheres com câncer... |14

vaginal vem se elevando, que as dificuldades para essa adesão abrangem a vergonha diante do uso e formato da prótese peniana, o desconforto sentido no momento da introdução do dispositivo no canal vaginal e a crença de que a manutenção da relação sexual é suficiente.

Quanto aos achados nas perguntas abertas, as narrativas, reunidas na categoria "Cuidados orientados e adotados pelas mulheres em braquiterapia pélvica", revelaram a diversidade de práticas de autocuidado. Ao comparar os achados das comunicações das participantes com as orientações das enfermeiras do CEPON, ${ }^{16}$ pode-se identificar que muitos cuidados orientados não foram citados pelas mulheres.

A ansiedade que algumas participantes sentem diante de uma entrevista pode ter influenciado esse achado ou as narrativas podem representar a escolha das mulheres de eleição de cuidados que desejam seguir, independente da recomendação profissional. Essa escolha configura uma prática comum das pessoas na definição dos seus itinerários de cuidados. Porém, os resultados também sugerem que pode haver necessidade de revisão da padronização das orientações de enfermagem e da complementação destas por material educativo. A revisão da prática de enfermagem e a elaboração de material educativo podem, por exemplo, contribuir para eliminação das queixas, no tocante à verbalização de uma participante que não recebeu algumas instruções por parte das enfermeiras, como registrado nos resultados deste estudo, na categoria “Cuidados não orientados pelos profissionais da saúde”.

Registra-se que as principais recomendações de cuidados às mulheres em braquiterapia pélvica, apontadas pelos profissionais do CEPON e por outros trabalhos, compreendem orientações sobre a anatomia dos órgãos genitais femininos, a braquiterapia e seus efeitos colaterais, cuidados para prevenir ou minimizá-los, agendamento das consultas de seguimento com enfermeiros, fisioterapeuta, ginecologista, oncologista, radioterapeuta, aconselhamentos sexuais e psicológicos, avaliação por psicólogo e/ou sexólogo, quando necessário. ${ }^{6,8,16-17}$ Esses cuidados não foram citados pelas participantes. 
Para prevenção e controle das sequelas imediatas ou tardias da braquiterapia, recomendam-se às mulheres desuso de cremes vaginais não indicados pela equipe de saúde; comunicação das alterações percebidas/sentidas pela mulher à equipe de saúde; retirada dos pelos pubianos antes do início do tratamento; jejum de 8 horas (antes do procedimento) para mulheres submetidas ao tratamento sob indução anestésica; presença de acompanhante; ingesta hídrica de 2,5 a 3 litros de líquido por dia; adoção de alimentação saudável; cuidados com a pele e as mucosas; avaliação e controle da dor; orientações sobre banho de sol, mar e piscina..$^{6,8,16-19}$ Desses cuidados, foram apenas citados pelas participantes a comunicação das alterações percebidas e a ingesta hídrica recomendada.

Cuidados relacionados à prevenção da inflamação vaginal e da estenose vaginal também são recomendado, tais como uso de ducha ginecológica com chá de camomila uma vez ao dia; dilatação vaginal com uso do pênis de silicone ou dilatador vaginal, em média, duas a quatro vezes por semana, por 5 a 10 minutos, iniciando esses exercícios após o término da braquiterapia, quando ocorrer a redução da resposta inflamatória; retorno à atividade sexual cerca de um mês após o término da braquiterapia, quando houver a redução da resposta inflamatória; uso de lubrificante vaginal e preservativo durante a relação sexual e/ou dilatação vaginal, por tempo indeterminado, podendo a frequência de uso dos exercícios de dilatação ser reduzida, caso a mulher mantenha relações sexuais eficazes; uso de tampões de vaselina (tampões cobertos de vaselina) inseridos durante a noite no canal vaginal, duas a três vezes por semana e durante pelo menos 9 a 12 meses após término do tratamento.

Ainda são recomendados o acompanhamento das alterações emocionais e sexuais relacionadas com a menopausa precoce e infertilidade, a avaliação e a classificação da estenose vaginal..$^{6,8,16-19} \mathrm{~A}$ maioria dos cuidados, aqui apresentados, foi revelada pelas participantes, o que retrata a maior preocupação com a prevenção da estenose vaginal ou, ainda, que esse cuidado pode estar sendo destacado nas orientações de enfermagem e da fisioterapia. 
Práticas de autocuidado e os efeitos colaterais imediatos em mulheres com câncer... | 16

Evidencia-se a necessidade de revisar a abordagem das mulheres em braquiterapia no cenário do estudo para aquisição de conhecimentos para as boas práticas de autocuidado e de mudanças do processo de educação em saúde desenvolvido pelos profissionais. Ainda, comparando os cuidados orientados pelas enfermeiras do $\mathrm{CEPON}^{19} \mathrm{com}$ as recomendações da literatura científica, ${ }^{8,9,20}$ identificou-se que a maioria compõe a prática profissional. As instruções não realizadas compreendem o uso do tampão com vaselina, orientações sobre a infertilidade e menopausa precoce e os encaminhamentos para sexólogos. Por conseguinte, sugere-se a inclusão dos mesmos na atenção em saúde prestada.

Quanto aos efeitos colaterais revelados pelas participantes, apresentados na categoria "Efeitos colaterais percebidos por mulheres em braquiterapia pélvica”, eles endossam a necessidade de cuidados preventivos e de ações profissionais para redução das disúrias, alterações intestinais e lesões na pele e mucosa anal, retal e vaginal. Destaca-se que as consequências relatadas por elas se relacionam às reações imediatas, pois, como as mesmas foram entrevistadas cerca de 45 dias após o término da braquiterapia, os impactos tardios não foram investigados. Os achados desta investigação, no que se refere aos efeitos colaterais que compõem a referida categoria temática, assemelham-se às evidências de outros estudos, ${ }^{11,21-23}$ exceto no que tange às alterações gástricas, que não foram citadas pelas participantes.

Pesquisa aponta que $21,2 \%$ das mulheres em braquiterapia pélvica apresentam toxicidades, $17,3 \%$ no trato gastrointestinal e $10 \%$ geniturinárias, e que a taxa de toxicidades estimada em três anos é de 24,2\%. A duração média do acompanhamento foi de 37 meses (variação de 3-146 meses). ${ }^{21}$ Os fatores associados às toxicidades foram o menor índice de massa corporal, a raça branca, o tabagismo ativo durante o tratamento, diabetes, tamanho dos ovóides utilizados na radiação e duração do tratamento. ${ }^{11,21}$

Estudo de revisão que inclui 87 publicações e que analisou a toxicidade imediata e tardia de mulheres submetidas à braquiterapia apresenta os seguintes graus de toxicidades agudas: 
sistema geniturinário: Grau 2: 1-54\%, Grau 3: 1-3\% e Grau 4: 0\%; toxicidade vinculada à quimiorradiação: Grau 2: 2-53\%, Grau 3: 1-3\% e Grau 4: 0\%. A toxicidade aguda gastrointestinal: Grau 2: 15-48\%, Grau 3: 3-11\% e Grau 4: 5\%; toxicidade vinculada à quimiorradiação: Grau 2: 562\%, Grau 3: $1-25 \%$ e Grau 4: 1-2\%. Ainda aponta que os efeitos agudos se caracterizam por sintomas geniturinários, tais como cistite, uretrite, hematúria, disúria, aumento do risco de infecções do trato urinário; e sintomas gastrointestinais, como risco de sangramento, dor, muco, hábitos intestinais irregulares, intolerâncias alimentares, náuseas e vômitos. ${ }^{22}$

Outra investigação, desenvolvida no território nacional, corrobora com esses achados afirmando que as toxicidades imediatas mais frequentes são as radiodermites e as que ocorrem nos sistemas gastrintestinal e geniturinário. Ainda aponta que o estado geral de saúde apresenta correlação com a faixa etária. ${ }^{23}$ Esses efeitos colaterais imediatos e os que devem surgir posteriormente, na fase tardia, alteram significativamente o modo de viver dessas mulheres. Nessa perspectiva, o apontado nas evidências científicas precisa ser visualizado no cotidiano do viver dessas mulheres. A urgência urinária e intestinal, a dor para urinar, a dor e o sangramento no canal vaginal ou durante a relação sexual alteram as relações sociais e sexuais, os papéis sociais, o modo como a mulher se vê e a qualidade de vida.

Pesquisa desenvolvida na África, que avaliou a qualidade de vida das mulheres, evidencia que, apesar de uma melhora no estado de saúde global, o câncer de colo do útero e seu tratamento tiveram uma influência negativa na qualidade de vida das mulheres e ocasionaram mudanças em todos os domínios de suas vidas. O funcionamento social foi o mais afetado e não melhorou, significativamente, ao longo do tempo. Durante o tratamento, as dificuldades financeiras influenciaram negativamente a qualidade de vida, como também a frequência urinária e a insônia presentes, sendo que essas sintomatologias iniciaram antes do diagnóstico e permaneceram mesmo após o término do tratamento; além do edema nos membros inferiores. ${ }^{24}$ O edema, em geral, é resultante de ressecções cirúrgicas de cadeias linfáticas ou de 
Práticas de autocuidado e os efeitos colaterais imediatos em mulheres com câncer... | 18

alterações ocasionadas nessas cadeias linfáticas pela radiação ionizante ou, ainda, pela neuropatia que pode surgir como toxicidade resultante da quimioterapia ou quimiorradiação. Nesse contexto, é fundamental que o enfermeiro assuma um papel que integre a realização de cuidados e de educação em saúde para o autocuidado como instrumento essencial no tratamento das mulheres com câncer ginecológico em braquiterapia.

A realização da consulta de enfermagem no contexto da braquiterapia permite maior interação do profissional, melhor tomada de decisão e compreensão do tratamento, por parte das pacientes, o que influencia a adoção das práticas de autocuidado e melhor estado de saúde desejado, ${ }^{9,25-26}$ colaborando para a diminuição dos medos, das ansiedades e dos desconhecimento. ${ }^{27}$ A desinformação configura fonte geradora de medo e insegurança, prejudiciais à saúde psicológica das mulheres e que podem contribuir negativamente para a corresponsabilização em saúde. ${ }^{28}$ Constatou-se que cabe ao enfermeiro na consulta de enfermagem minimizar o medo, a insegurança e os desconhecimentos.

Por fim, percebe-se que o papel de cada profissional da equipe multiprofissional é imprescindível para a melhor qualidade de vida da mulher com câncer ginecológico em braquiterapia, uma vez que a soma das atuações, incluindo avaliação e orientações, amplia a gama de cuidados que devem ser ofertados durante o tratamento. Concluído o tratamento, os controles em saúde com os médicos assistentes são essenciais, contudo o seguimento continuado em curtos intervalos de tempo com o fisioterapeuta contribui para a qualidade de vida da mulher, destacandose a saúde sexual, prevenção de linfedemas, controle das alterações intestinais e urinárias.

Como limitação da pesquisa, aponta-se a realização da investigação em um único cenário. Todavia, como se trata de uma abordagem qualitativa, entende-se que o método é replicável e os resultados aqui apresentados são relevantes e revelam o que, comumente, observa-se na prática clínica dos profissionais atuantes nessa área de interesse. 


\section{Conclusão}

Os efeitos colaterais revelados pelas mulheres em suas narrativas incluem as alterações gastrointestinais, geniturinárias, na pele e mucosa (vaginal, anal e retal). Quanto aos cuidados adotados pelas mulheres, destacou-se a prevenção da estenose vaginal (uso de dilatador vaginal no formato de pênis de silicone, lubrificante vaginal, higienização e guarda da prótese), seguida dos cuidados relacionados ao controle da inflamação vaginal (uso de lavagem vaginal com chá de camomila) e da disúria (ingestão de chá de quebra-pedra).

Os resultados deste estudo permitiram a elaboração de uma cartilha educativa para complementação da educação em saúde realizada pelas enfermeiras em suas consultas no CEPON. Além disso, o planejamento de enfermagem para a consulta está sendo discutido pela equipe.

Os resultados ainda reforçaram o papel relevante da consulta de enfermagem e do fisioterapeuta para melhor atenção oncológica e qualidade de vida durante e após o término do tratamento. Considerando a natureza do estudo, os efeitos colaterais tardios não foram investigados, assim, recomenda-se que novas investigações devam ser realizadas para contemplar esse contexto.

\section{Referências}

1. Funston G, O'Flynn H, Ryan NAJ, Hamilton W, Crosbie EJ. Recognizing gynecological cancer in primary care: risk factors, redflags, and referrals. Adv Ther. 2018; 35(4):577-89. doi: 10.1007/s12325-0180683-3

2. Soo-Young L, Kim E, Kim HS, Koo YJ, Lee DH. Clinical and histopathologic analysis of gynecological cancer: a single institute experience over 7years. Yeungnam Univ J Med. 2020;37(3):179-85. doi: 10.12701/yujm.2019.00451

3. Ferlay J, Ervik M, Lam F, Colombet M, Mery L, Piñeros M, et al. Global Cancer Observatory: Cancer Today [Internet]. Lyon (FR): International Agency for Research on Cancer; 2018 [cited 2019 Aug 14]. Available from: https://gco.iarc.fr/today

4. Chargari C, Deutsch E, Blanchard P, Gouy S, Martelli H, Guérin F, et al. Brachytherapy: an overview for clinicians. CA Cancer J Clin. 2019 Sep; 69(5):386-401. doi: 10.3322/caac.21578 
Práticas de autocuidado e os efeitos colaterais imediatos em mulheres com câncer... | 20

5. Mahantshetty U, Gudi S, Singh R, Sasidharan A, Sastri SC, Gurram L, et al. Indian Brachytherapy Society Guidelines for radiotherapeutic management of cervical cancer with special emphasis on highdose-rate brachytherapy. J Contemp Brachytherapy. 2019; 11(4):293-306. doi: 10.5114/jcb.2019.87406

6. Nascimento FC, Deitos J, Luz CM. Comparação da disfunção do assoalho pélvico com função sexual e qualidade de vida em sobreviventes ao câncer ginecológico. Cad Bras Ter Ocup. 2019; 27(3):628-37. doi: 10.4322/2526-8910.ctoao 1640

7. Morris L, Do V, Chard J, Brand AH. Radiation-induced vaginal stenosis: current perspectives. Int J Womens Health. 2017; 9:273-9. doi: 10.2147/IJWH.S106796

8. Matos SRL, Cunha MLR, Podgaec S, Weltman E, Centrone AFY, Mafra ACCN. Consensus for vaginal stenosis prevention in patients submitted to pelvic radiotherapy. PLoS One. 2019; 14(8):e0221054. doi: 10.1371/journal.pone.0221054

9. Bakker RM, Mens JWM, Groot HE, Tuijnman-Raasveld CC, Braat C, Hompus WCP, et al. A nurse-led sexual rehabilitation intervention after radiotherapy for gynecological cancer. Support Care Cancer. 2017; 25:729-37. doi: 10.1007/s00520-016-3453-2

10. Long D, Friedrich-Nel HS, Joubert G. Brachytherapy for cervical cancer: guidelines to facilitate patient-centred care in a multidisciplinary environment. S Afr J Gynaecol Oncol. 2016; 8(2):27-33. doi: $10.1080 / 20742835.2016 .1212968$

11. Romano KD, Hill C, Trifiletti DM, Peach MS, Horton BJ, Shah N, et al. High dose-rate tandem and ovoid brachytherapy in cervical cancer: dosimetric predictors of adverse events. Radiat Oncol. 2018; 13(129):1-10. doi: 10.1186/s13014-018-1074-2

12. Clandinin DJ. Engaging in Narrative Inquiry. WalnutCreek, CA: Left Coast Press; 2013.

13. Bardin L. Análise de conteúdo. São Paulo: Edições 70; 2011.

14. Silva AAL, Rosa LMR, Schoeller SD, Radünz V, Martins MM, Martins HIV, et al. Sociodemographic and clinical profile of women with cancer in the genital tract who underwent radiation therapy. Cogitare Enferm. 2019; 24:e58467. doi: 10.5380/ce.v24i0.58467

15. Valakh V, Coopey BC. Factors associated with duration of overall treatment time for cervical cancer treated with definitive chemoradiotherapy. Cureus. 2019; 11(10):e5951. doi: 10.7759/cureus.5951

16. Bedell SL, Goldstein LS, Goldstein AR, Goldstein AT. Cervical Cancer Screening: past, present, and future. Sex Med Rev. 2020;8(1):28-37. doi: 10.1016/j.sxmr.2019.09.005

17. Law E, Kelvin JF, Thom B, Riedel E, Tom A, Carter J, et al. Prospective study of vaginal dilator use adherence and efficacy following radio therapy. Radiother Oncol. 2015; 116:149-55. doi: 10.1016/j.radonc.2015.06.018 
18. Dias M, Custodio LL, Patricio JJS, Bragante KC, Toriy AM, Souza CPF, et al. Efectiveness of the use of silicone penile prosthesis in the prevention of vaginal stenosis after high dose rate brachytherapy. J Sex Med. 2018; 15(7 Suppl 3):S345-6. doi: 10.1016/j.jsxm.2018.04.492

19. Pessi MR, Feurchutte KK, Rosa LM, Hammerschmidt KSA, Radünz V, Alvarez AM. Prevention of vaginal stenosis after brachytherapy: nursing intervention. Rev Enferm UFPE On Line [Internet]. 2016 [cited 2019 Aug 14]; 10(9):3495-502. Available from: https://periodicos.ufpe.br/revistas/revistaenfermagem/article/view/11433

20. The Ottawa Hospital. Patient information: a guide to your HDR brachytherapy for cancer of the cervix. Ottawa (CA): The Ottawa Hospital; 2016 [cited 2019 Aug 16]. Available from: http://www.ottawahospital.on.ca/en/documents/2017/01/cp85b-hdr-brachy-therapy-of-cervix-englishapril-2016.pdf/

21. Rubinsak LA, Kang L, Fields EC, Carter JS, McGuire WP, Temkin SM. Treatment-related radiation toxicity among cervical cancer patients. Int J Gynecol Cancer. 2018; 28(7):1387-93. doi: 10.1097/IGC.0000000000001309

22. Mayadev J, Viswanathan A, Liu Y, Li CS, Albuquerque K, Damato AL, et al. American Brachytherapy Task Group Report: a pooled analysis of clinical outcomes for high-dose-rate brachytherapy for cervical cancer. Brachytherapy. 2017;16(1):22-43. doi: 10.1016/j.brachy.2016.03.008

23. Silveira CF, Regino PA, Soares MBO, Mendes LC, Elias TC, Silva SR. Quality of life and radiation toxicity in patients with gynecological and breast cancer. Esc Anna Nery Rev Enferm. 2016; 20(4):e20160089. doi: 10.5935/1414-8145.20160089

24. Sabulei C, Maree JE. An exploration into the quality of life of women treated for cervical cancer. Curationis. 2019;42(1):e1-9. doi: 10.4102/curationis.v42i1.1982

25. Rosenblatt E, Zubizarreta E, editors. Radiotherapy in cancer care: facing the global challenge. Vienna: International Atomic Energy Agency; 2017 [cited 2020 Mar 27]. Available from: https://wwwpub.iaea.org/MTCD/Publications/PDF/P1638_web.pdf

26. Marcon C, Argente C, Metelski FK, Schorr V, Adamy EK. Characterization of patients in radiotherapical treatment from the nursing consultation. Rev Enferm UFSM. 2017;7(1):61-8. doi: $10.5902 / 2179769224914$

27. Araújo CRG, Rosas AMMTF, Menezes HF, Pinto ACS, Rodrigues BMRD. The phenomenon lived by women in nursing consultation in the gynecological brachytherapy. Texto Contexto Enferm. 2017; 26(2):e00140016. doi: 10.1590/0104-07072017000140016

28. Duarte EB, Rosa LM, Radünz V, Dias M, Silva RH, Lunardi F, et al. Women in pelvic brachiterapy: (un)knowledge and professional care as meaning. Cogitare Enferm. 2020; 25:e68406. doi: 10.5380/ce.v25i0.68406 
Práticas de autocuidado e os efeitos colaterais imediatos em mulheres com câncer... | 22

Editora Científica Chefe: Cristiane Cardoso de Paula

Editora associada: Silvana Bastos Cogo

Fomento / Agradecimento: Não possui.

\section{Autor correspondente}

Luciana Martins da Rosa

E-mail: luciana.m.rosa@ufsc.br

Endereço: R. Eng. Agrônomo Andrei Cristian Ferreira, 570 - Trindade, Florianópolis - SC - Brasil. Centro de

Ciências da Saúde, bloco I, sala 412.

CEP: 88040-535.

\section{Contribuições de Autoria}

\section{1 - Rosimeri Helena da Silva}

Concepção ou desenho do estudo/pesquisa, análise e/ou interpretação dos dados, revisão final com participação crítica e intelectual no manuscrito.

\section{2 - Luciana Martins da Rosa}

Concepção ou desenho do estudo/pesquisa, análise e/ou interpretação dos dados, revisão final com participação crítica e intelectual no manuscrito.

\section{3 - Mirella Dias}

Revisão final com participação crítica e intelectual no manuscrito.

\section{4 - Nádia Chiodelli Salum}

Revisão final com participação crítica e intelectual no manuscrito.

\section{5 - Ana Inêz Severo Varela}

Revisão final com participação crítica e intelectual no manuscrito.

6 - Vera Radünz

Revisão final com participação crítica e intelectual no manuscrito.

\section{Como citar este artigo}

Silva RH, Rosa LM, Dias M, Salum NC, Varela AIS, Radünz V. Práticas de autocuidado e os efeitos colaterais imediatos em mulheres com câncer ginecológico em braquiterapia. Rev. Enferm. UFSM. 2021 [Acesso em: Anos Mês Dia]; vol.11 e35: 1-22. DOI: https://doi.org/10.5902/2179769248119 